\title{
Playability: How to Identify the Player Experience in a Video Game
}

\author{
J.L. González Sánchez, N. Padilla Zea, and F.L. Gutiérrez \\ Software Engineering Department, University of Granada, E-18071, Spain \\ \{joseluisgs, npadilla, fgutierr\}@ugr.es
}

\begin{abstract}
Nowadays Video Games occupy a privileged position in the leisure and entertainment market, representing the fastest-growing industry globally. In this paper we will analyse how, in video game development, Usability alone is not sufficient to achieve the optimum Player Experience. We argue that the concept of Usability needs broadening and deepening, to embrace further attributes and properties that identify and describe the Player Experience. We present our proposed means of defining Playability, its attributes and properties and how associate them with the different elements of a video game.
\end{abstract}

Keywords: Playability, Usability, User Experience, Video Games.

\section{Introduction}

Throughout history, humans have had the capacity to manage their own leisure time, this being a significant driver in cultural development. Nowadays, video games and entertainment systems collectively make up the biggest industry in terms of turnover, more so than music and cinema. From this we can deduce that video games have become the preferred game of choice, exerting significant social and cultural influence over children, teens and adults [1]. In this paper we analyse why Usability is therefore not sufficient to describe the full User Experience in relation to video games. Secondly, we present a definition of Playability, its attributes and properties, to characterise and measure the Player Experience with these kinds of systems. Finally, we introduce the notion of Facets of Playability that will allow us to study Playability easily across the different video game elements, testing and analysing each attribute and its properties throughout the video game development process.

\section{Playability in Entertainment Systems}

When a Desktop System (DS) such as a word processor, is developed, the main objective is that users can execute a set of tasks in a predetermined context, for example working in an office. Usability is a measure of product use whereby users achieve concrete objectives in varying degrees of effectiveness, efficiency and satisfaction, within a specific context of use [2]. The User Experience (UX) [3] is facilitated by the Usability. It has two principal points of view, which characterize it: process (of use) and product. Video Games can be considered a 'special' interactive system, they have 
a very specific objective: to make the player feel good when playing it. We propose that analysing the quality of a video game purely in terms of its Usability is not sufficient - we need to consider not only functional values but also non-functional values, given the specific properties of video games. In other words, the Player Experience (PX) could be much more complex than the UX. It entails to extend and complete formally the UX characteristics with players' dimensions (user and group) using a broad set of attributes and properties in order to identify and measure the experience of players playing a video game, $P X$. These properties indicate to us whether a game is 'playable' or not - that is, they will identify the Playability of the video game.

Although Playability is a live topic in the scientific community, it has been studied from different points of view and with different objectives without consensus on its definition or the attributes or properties to characterize it: Playability as only Usability in video games context $[4,5,6]$ or in the quality of game elements $[7,8]$.

We define Playability as: ' $a$ set of properties that describe the Player Experience using a specific game system whose main objective is to provide enjoyment and entertainment, by being credible and satisfying, when the player plays alone or in company'. Playability is characterised by attributes that exist in Usability but that have different meanings in video game context (Fig. 1). Playability represents the degree to which specified users can achieve specified goals with effectiveness, efficiency and specially satisfaction and fun in a playable context of use. We propose a set of seven attributes to characterise Playability and some examples of properties of Playability, to subsequently measure them:

Playability: The degree to which specified users can achieve specified goals with effectiveness, efficiency and specially satisfaction and fun in a playable context of use. Example: The Legend of Zelda

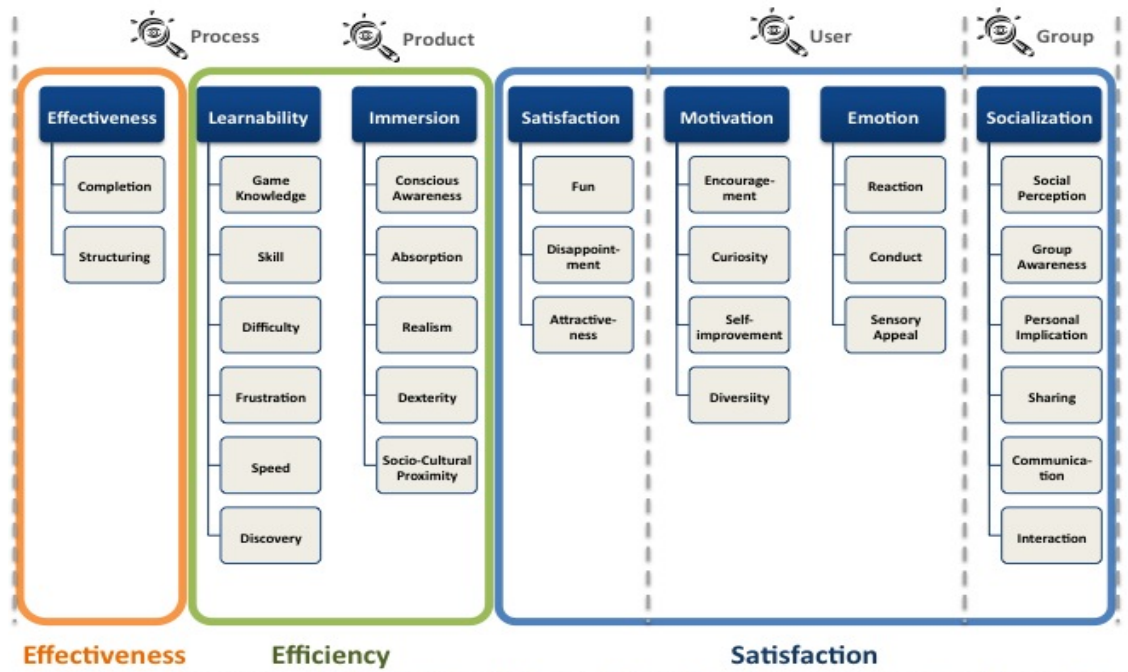

Usability - ISO 9241-11: The extent to which a product can be used by specified users to achieve specified goals with effectiveness, efficiency and satisfaction in a specified context of use. Example: Word Processor

Fig. 1. Playability Model: attributes and properties to measure it 
Satisfaction: We define this as the gratification or pleasure derived from playing a complete video game or from some aspect of it. We characterise it using the following properties: Game Fun, Contents Disappointment and Attractiveness. Learnability: We define this as the player's capacity to understand and master the game's system and mechanics (objectives, rules, how to interact with the video game, and so on). We propose the following properties to characterise it: Game Knowledge, Player Skill, Game Difficulty, Player Frustration, Speed of Learning and Discovery Techniques. Effectiveness: We define this as the time and resources necessary to offer players a fun and entertaining experience whilst they achieve the game's various objectives and reach the final goal. We identify Effectiveness as having the following properties: Game Completion and the Structuring of the Game Resources. Immersion: We define this as the capacity of the video game contents to be believable, such that the player becomes directly involved in the virtual game world. To characterise Immersion we propose the following properties: Conscious Awareness, Absorption in game, Game Realism, Control Dexterity and Socio-Cultural Proximity with the game. Motivation: We define this as the set of game characteristics that prompt a player to realise specific actions and continue undertaking them until they are completed. We characterise Motivation as having the following properties: Encouragement Techniques, Curiosity about the game, Player Self-Improvement and Diversity of game resources. Emotion: This refers to the player's involuntary impulse in response to the stimulus of the video game that induces feelings or a chain reaction of automatic behaviours. We characterise Emotion as having the following properties: Player Reaction, Game Conduct and Sensory Appeal for game elements. Socialization: We define this as the set of game attributes, elements and resources that promote the social dimension of the game experience in a group scenario. We propose that Socialization has the following properties: Social Perception, Group Awareness, Personal Implication, the Sharing of the Social Resources, Communication Techniques and Interaction Rules of the socialization game.

Playability analysis is a very complex process due to the different perspectives that we can use to analyse the various parts of video game architecture. In this work, we propose a classification of these perspectives based on six Facets of Playability. Each facet allows us to identify the different attributes and properties of Playability that are affected by the different elements of video game architecture [9]. The first facet is Intrinsic Playability: this is the Playability inherent in the nature of the video game itself and how it is presented to the player. Mechanical Playability: it is related to the quality of the video game as a software system. Interactive Playability: this is associated with player interaction and video game user interface development. Artistic Playability: this facet relates to the quality of the artistic and aesthetic game elements and how these elements are executed in the video game. Intrapersonal Playability or Personal Playability: This refers to the individual outlook, perceptions and feelings that the video game produces in each player when they play, and as such has a high subjective value. Interpersonal Playability or Social Playability: This refers to the feelings and perceptions of users, and the group awareness that arise when a game is played in company, be it in a competitive, cooperative or collaborative way.

The overall Playability of a video game, then, is the sum total of values across all attributes in the different Facets of Playability. It is crucial to optimise Playability across the different facets in order to guarantee the best Player Experience. 


\section{Conclusions and Future Work}

In this paper we have presented video games as special interactive systems developed to entertain the user, concluding that Usability alone is an insufficient measure for determining the full Player Experience. We have presented the concept of Playability outlining the attributes that characterise it and their properties, in order to measure and guarantee an optimum Player Experience. To facilitate the analysis of Playability, we have proposed the Facets of Playability to study every property in each attribute in order to identify the elements necessary to achieve overall Playability in different video games. Playability must be taken into account in every phase of the game development, in order to, amongst other things, anticipate any unexpected or negative results for the developer and guarantee a high quality of playability and improve the Player Experience in the final product.

Currently we are designing a conceptual model of a video game which will enable us to specify and analyse Playability characteristics in the design phase, and to incorporate Playability techniques into software patterns, style guides and heuristic techniques, thus ensuring optimum Playability of the end-product. We are also adapting techniques used in Usability Engineering and User-Centred Design in order to include Playability in a quality model to enhance the Player Experience throughout the different phases of video game development.

Acknowledgments. This research is financed by: the Spanish International Commission for Science and Technology (CICYT); the DESACO Project (TIN2008-06596C02-2); and the F.P.U. Programme of the Ministry of Science and Innovation, Spain.

\section{References}

1. Provenzo, E.: Video kids. Harvard University Press, Cambridge (1991)

2. ISO 9241-11: Guidance on Usability, also issued by the International Organization for Standardization (1998)

3. Hassenzahl, M., Tractinsky, N.: User Experience - A Research Agenda. Behaviour and Information Technology 25(2), 91-97 (2006)

4. Federoff, M.: Heuristic and Usability Guidelines for the Creation and Evaluation of Fun Video Games. Master Thesis, Department of Telecommunications, Indiana University (2002)

5. Desuvire, H., Capñan, M., Toth, J.: Using Heuristic to Evaluate The Playability in Games. In: CHI 2004. ACM, New York (2004)

6. Fabricatore, C., Nussbaum, M., Rosas, R.: Playability in Action Video Games: A Qualitative Design Model. Human-Computer Interaction 17, 311-368 (2002)

7. Rollings, A., Morris, D.: Game Architecture and Design. Ed. New Riders Games (2003)

8. Salen, K., Zimmerman, E.: Rules of Play: Game Design Fundamentals. Ed. The MIT Press, Cambridge (2003)

9. González Sánchez, J.L., Padilla Zea, N., Gutiérrez, F.L.: From Usability to Playability: Itroduction to the Player-Centred Video Game Development Procces. In: Procedings of HCI International (Forthcoming 2009) 